Schleimhautmelanom zusätzlich auf cKIT, so Hauschild. Vor allem die c-KITHot-Spot-Mutationen L576P und K642E in Exon 11 und 13 ließen sich mit dem cKIT-Kinaseinhibitor Imatinib gezielt therapieren. „Patienten mit diesen Mutationen haben eine Chance von fast $50 \%$ auf eine Remission“, berichtete Hauschild.

NRAS-Mutationen treten bei etwa $20 \%$ der Melanome auf. Bislang gab es keinen Inhibitor gegen dieses Protoonkogen, das eine wichtige Rolle in der Signalkaskade in Richtung Proliferation spielt. Der MEK-Inhibitor Binimetinib jedoch führte bei Patienten mit fortgeschrittenem NRAS-mutiertem Melanom $\mathrm{zu}$ einem deutlich besseren medianen progressionsfreien Überleben im Vergleich zu einer Dacarbazin-Therapie (2,8 vs. 1,5 Monate; Hazard Ratio $0,62, \quad 95 \%$-Konfidenzintervall $0,47-$ 0,$80 ; \mathrm{p}<0,001$ ) [Dummer R et al. ASCO. 2016;Abstr 9500]. Allerdings ohne in ein besseres Gesamtüberleben zu münden (11,0 vs. 10,1 Monate). Ein neuer Standard wird Binimetinib daher Hauschild zufolge wohl nicht; zu prüfen wäre aber, ob es in Kombination mit einem BRAFInhibitor von Nutzen sei.

\section{Strategien gegen \\ Resistenzentwicklung}

Patienten mit BRAF-mutierten Tumoren lassen sich mit BRAF-Kinaseinhibitoren wie Vemurafenib oder Dabrafenib erfolgreich behandeln - das mediane Überleben verdoppelt sich gegenüber der Chemotherapie. Allerdings entwickeln sich unter Monotherapien häufig Resistenzen, weshalb in der aktuellen Leitlinie immer eine Kombinationstherapie aus BRAF- und MEK-Inhibitoren (MEKi) empfohlen wird.

Zusätzlich werden mehrere Strategien gegen die Resistenzentwicklung erprobt. Etwa die Intervalltherapie, in der BRAF/MEKi in Blöcken von acht oder zwölf Wochen gegeben werden und anschließend eine Pause erfolgt. Bereits durchgesetzt hat sich laut Hauschild, dass man nach einem Progress die Kombinationstherapie fortführt, einzelne Herde jedoch mit Strahlentherapie behandelt. „Spannend sind momentan laufende Studien, welche die Immuntherapie mit BRAF/MEKi kombinieren. Diesen Ansatz halte ich für sehr

\title{
Kutane Nebenwirkungen unter Checkpointinhibition
}

Nachdem die Immuntherapie mit Checkpointinhibitoren nicht nur bei Melanompatienten Einzug gehalten hat, sondern sich zunehmend auch bei Patienten mit anderen Krebserkrankungen etabliert, wird der Blick auf die Nebenwirkungen - auch an der Haut - immer wichtiger. „Kutane Nebenwirkungen treten unter der Checkpointblockade zwar häufig auf, erreichen aber selten höhere Schweregrade", erläuterte Ralf Gutzmer, Hannover, im Rahmen der Fortbildungswoche für praktische Dermatologie und Venerologie 2016. Bei etwa $10 \%$ der Melanompatienten unter Immuntherapie käme es zu einer Vitiligo, so Gutzmer weiter. Dabei sei das Auftreten der Vitiligo unter PD1 („programmed cell death protein 1“)-Blockade mit einem besseren Ansprechen assoziiert [Hua C et al. JAMA Dermatol. 2016;52(1):45-51]. Er wies auch darauf hin, dass diese Patienten zudem weitere Autoimmunerkrankungen entwickeln könnten, wie z. B. Typ1-Diabetes. Eine andere Nebenwirkung, die beobachtet werde, sei Rash. In der Regel handele es sich um makulopapulöse Exantheme, die mit topischen Steroiden gut zu behandeln seien. Bei schweren Verläufen seien systemische Steroide indiziert, möglicherweise sei auch eine Therapiepause erforderlich.

Auch therapiebegleitender Juckreiz werde beobachtet. Dieser ist laut Gutzmer häufig mild und lässt sich gut mit rückfettender Hautpflege, Antihistaminika und bei Ekzematisierung mit topischen Steroiden therapieren.

In Einzelfällen, so Gutzmer, komme es unter der Anti-PD-1-Therapie auch zu entzündlichen Hauterkrankungen, etwa Psoriasis, bullösen Dermatosen oder Lichen ruber mucosae. In diesen Fällen sind Gutzmer zufolge eine systemische Therapie mit Steroiden und eine Unterbrechung der PD-1-Inhibition ratsam. Bei vorbestehenden Erkrankungen aus diesem Formenkreis sei immer eine sorgfältige Nutzen-Risiko-Analyse im Rahmen der Tumorkonferenz erforderlich, betonte Gutzmer. Marion Hofmann-Aßmus

vielversprechend“, so Hauschild. Seiner Meinung nach wird es zukünftig darum gehen, die verfügbaren Substanzklassen intelligent zu verknüpfen.

\section{S2k-Leitlinie Mycosis fungoides}

Die Mycosis fungoides ist ein primär kutanes T-Zell-Lymphom. Da die Mycosis fungoides in Phasen abläuft, sind eine exakte Diagnose sowie die Berücksichtigung der Vorbehandlung und des Tumorstadiums wichtig. Dies betonte Claus-Detlef Klemke, Karlsruhe. Unabhängige Marker für eine schlechte Prognose bei fortgeschrittener Mycosis fungoides (Stadium IIB-IV) oder SézarySyndrom identifizierte kürzlich das Cutaneous Lymphoma International Consortium (CLIC) [Scarisbrick J et al. J Clin Oncol. 2015;33(32):3766-73]: Stadium IV, Alter > 60 Jahre, großzellige Transformation in der Haut, erhöhte LDH (L-Lactatdehydrogenase). Die 5-Jahres-Überlebensrate liege bei Patienten mit niedrigem Risiko (0-1 Risikofaktor) bei $68 \%$, bei mittlerem Risiko ( 2 Risikofaktoren) bei $43 \%$ und bei hohem Risiko (3-4 Risikofaktoren) bei 28\%. Klemke gab einen kurzen Einblick in das Update der S2k-Kurzleitlinie. Als
Erstlinientherapie der Mycosis fungoides im Stadium IA-IIA werden hautgerichtete Therapien empfohlen, wie etwa Steroide der Klasse III-IV oder PUVA (Psoralen/UV-A-Therapie). Einfacher durchzuführen als PUVA sei die Therapie mit Schmalspektrum UVB mit einer Wellenlänge von $311 \mathrm{~nm}$, die ebenfalls hervorragend funktioniere, so der Dermatologe.

Neu in der Zweitlinientherapie sei die Empfehlung für Mechlorethamin sowie für Acitretin als Alternative für Patienten, die kein Bexaroten erhalten können. In fortgeschrittenen Stadien stehen in der Erstlinie Kombinationen im Vordergrund: PUVA plus Interferon $a($ IFN- $\alpha$ ) oder oralem Bexaroten bzw. mit Radiotherapie. Im Stadium III werde die Kombination PUVA/UVB-311 nm empfohlenen, ggf. kombiniert mit IFN-a oder Bexaroten. In der Zweitlinie seien z.B. Pralatrexat oder der gegen CD30 gerichtete Antikörper Brentuximab vedotin neu hinzugekommen, so Klemke.

Marion Hofmann-Aßmus

Bericht von der 25. Fortbildungswoche für praktische Dermatologie und Venerologie vom 23. bis 29. Juli 2016 in München. 